\title{
Otium, mémoire du lieu et écriture de soi. Le cas
} Henry Brulard

Otium, Memory of Place and Writing of the Self. The Case of Henry Brulard

\section{Anna Karina Sennefelder}

\section{OpenEdition}

\section{Journals}

Édition électronique

URL : https://journals.openedition.org/recherchestravaux/809

DOI : 10.4000/recherchestravaux.809

ISSN : 1969-6434

Éditeur

UGA Éditions/Université Grenoble Alpes

Édition imprimée

Date de publication : 1 juin 2016

Pagination : $51-70$

ISBN : 978-2-84310-325-4

ISSN : 0151-1874

Référence électronique

Anna Karina Sennefelder, "Otium, mémoire du lieu et écriture de soi. Le cas Henry Brulard»,

Recherches \& Travaux [En ligne], 88 | 2016, mis en ligne le 01 janvier 2017, consulté le 29 octobre 2021.

URL : http://journals.openedition.org/recherchestravaux/809 ; DOI : https://doi.org/10.4000/

recherchestravaux.809 
Anna Karina Sennefelder

Université Albert-Ludwig de Fribourg-en-Brisgau, SFB iors «Muße»

\section{Otium, mémoire du lieu et écriture de soi. Le cas Henry Brulard}

\section{La relation traditionnelle entre l'idylle, l'otium et l'introspection}

La relation évidente entre l'otium, l'idylle et l'introspection narrative est issue d'une tradition culturelle et littéraire très ancienne selon laquelle le travail sur soi ne demande pas seulement un temps d'otium, ou bien de loisir ${ }^{2}$, mais aussi un lieu spécifique :

Avec le travail sur soi, on associe [depuis Horace] l'idée d'une vie retirée, d'une vie qui se passe dans la tranquillité d'une intimité à l'abri des stimuli extérieurs. À cet effort moral correspondait un lieu particulier, le locus amoenus, un lieu de plaisir, un espace de récréation en campagne, une atmosphère d'été agréable avec des jardins, des chemins de promenades, des sources et des monuments plaisants [...]. Cette

I. Le terme «idylle» est ici employé d'après la définition de M. Bakhtine, Esthétique et théorie du roman, trad. du russe D. Olivier, Paris, Gallimard, I987, p. 367-368. L'idylle amoureuse n'en est qu'un aspect, et elle suppose plus généralement un «micromonde» amoureux, laborieux ou familial, «limité dans l'espace», potentiellement d'une «durée infinie» et qui «se suffit à lui-même».

2. La terminologie française n'est pas tout à fait claire, comme le souligne M. Fumaroli. Pour lui, loisir est un quasi-synonyme d'otium, tandis que "l'oisiveté» et «les loisirs» en sont les adversaires : "Le loisir - en grec scholè [...] en allemand $M u ß e$ [...] est en dernière analyse [...] un principe classique d'art de vivre et de santé spirituelle, et non un temps de luxe gaspillé par des oisifs. ( (M. Fumaroli, «Préface. Plaidoyer pour le loisir et le regard de loisir», dans M. Fumaroli, D. Simon, J.-C. Darmon, G. Métayer [dir.], L’otium dans la République des lettres, Paris, Baudry, 20II, p. 9.) De plus, Fumaroli précise : "La différence entre loisir et loisirs, c'est que le loisir est un état de vacance intérieure, où l'on jouit d'abord de soi et des siens, alors que les loisirs supposent une attitude affairée, calculatrice et consommatrice [...] qui perpétue dans les heures de vacance et jusque dans l'amour tous les plis contractés dans le temps des affaires.» (M. Fumaroli, «Préface : Loisirs et loisir», dans M. Fumaroli, P.-J. Salazar, E. Bury [dir.], Le Loisir lettré à l'âge classique, Genève, Droz, I996, p. I4.) 
idylle constituait le modèle opposé d'un quotidien fatiguant où l'on n'arrivait pas à trouver de repos ${ }^{3}$.

Au premier abord, il semble facile de nommer les qualités de ce lieu, dans lequel un sujet se retire pour se consacrer à loisir à lui-même, parce qu'il s'agit de toute évidence d'un type de lieu qu'on peut rapporter sans problème au paradigme des «idylles», c'est-à-dire des lieux naturels et beaux, bien protégés, des " contre-mondes utopiques, libres de conflits qui sont marqués par l'unité, l'harmonie et la paix ${ }^{4}$. Dans un lieu de ce type, le sujet retiré peut se dévouer tranquillement à ses propres pensées et il y est, au sens d'une autosuffisance rousseauistes, renvoyé à lui-même.

On peut trouver un exemple précoce d'un tel lieu dans le Roman de la Rose. Le beau jardin y est non seulement gardé par une sæur de l'otium, Oiseuse ${ }^{6}$, mais le jardin constitue aussi le lieu où le héros, Amant, se réalise à travers son aventure amoureuse et plus encore à travers la narration de son histoire. Comme c'est Oiseuse qui lui ouvre le lieu de l'expérience et de la narration, elle apparait d'une part comme une figure positive qui permet au narrateur d'entrer dans l'espace de l'action, ce qui engendre le récit. D'autre part, elle est présentée comme une figure liminaire aux qualités et au comportement vicieux ${ }^{7}$. Oiseuse représente alors un spectre ambivalent qui oscille entre un otium bonum et une otiositas condamnable. Mais c'est surtout sa fonction allégorique de gardienne du jardin qu'il faut souligner : Oiseuse est, dans sa dimension positive qui est associée à la notion d'otium, favorable à la création et aux arts. C'est elle qui veille sur le lieu idyllique, qui permet à celui qui y entre d'y trouver un lieu d'autoréalisation et de création.

3. E. Straub, «Das Glück, das sich verweigert», dans M. Gemmel, C. Löschner (dir.), Ökonomie des Glücks. Muße, Müßiggang und Faulheit in der Literatur, Berlin, Ripperger \& Kremers, 20I4, p. I7-30, p. I8. (Je traduis.)

4. P. Ph. Riedl, "Die Kunst der Muße. Über ein Ideal in der Literatur um I800 », Publications of the English Goethe Society, nº 80, 20II, p. 26. (Je traduis.)

5. Il est bien connu que Rousseau a établi la dichotomie entre l'amour-propre, qui est motivé par les lois de la société, et l'amour de soi qui est naturel à l'homme, notamment dans son Discours sur l'origine et les fondements de l'inégalité parmi les hommes. Pourtant, M. Locke McLendon a récemment jeté un regard nouveau sur ces termes antithétiques (M. Locke McLendon, «Rousseau's Amour-propre. A Psychological Source of Civic Distrust in Liberal Societies", European Journal of Political Theory, n ${ }^{\circ}$ 3, 20I4, p. 34I-36I).

6. Oiseuse se présente elle-même à Amant et au lecteur dans les vers 598-606. Voir G. de Lorris, J. de Meung, Le Roman de la Rose, suivi de notes et d'un glossaire par P. Marteau, Nendeln/ Liechtenstein, Kraus Repr., I970, p. 4I.

7. K. Fallend montre dans son article toutes les qualités d'Oiseuse qui sont parfois contradictoires (K. Fallend, «Oiseuse zwischen courtoisie, Muße und Sünde: eine umstrittene Figur aus dem Roman de la Rose», Zeitschrift für romanische Philologie, n ${ }^{\circ}$ 26, 2010, p. 226-236). 
On trouve une autre corrélation célèbre entre un lieu, l'otium et l'introspection dans le chapitre viI des Essais de Montaigne qui s'intitule «De l'oisiveté». Dans ce traité très court, il y a également une réflexion sur les ambivalences de l'oisiveté. On ne peut discuter ici les variations sémantiques, ni la relation compliquée entre l'otium et l'oisiveté, qui évolue beaucoup, surtout au début du XIX ${ }^{e}$ siècle. Ce qui importe, c'est que, dans «De l'oisiveté», la retraite, l'isolement et l'étude de soi ne sont possibles que grâce à l'introduction d'une discipline sévère, parce que l'esprit oiseux, qui est livré à lui-même, se comporte comme un "cheval eschappé ${ }^{8}$ " et ne produit que des "chimères" et des "monstres fantasques ${ }^{9}$ ". Bien sûr, cette discipline est l'écriture elle-même. Chez Montaigne, elle sert d'outil de réglage à l'aide duquel le sujet essaie de reconquérir une souveraineté sur ses propres pensées. Montaigne rejoint avec son essai l'opposition traditionnelle entre une oisiveté favorable à l'introspection et à l'autonarration et une oisiveté qui y est défavorable, et il se présente comme un apologiste de l'oisiveté qui mène au bonheur et à une connaissance approfondie de soi-même. Certes, Montaigne lui-même ne pouvait pas vivre un otium cum litteris aussi tranquille que le chapitre vir le suggère, au moins dans une interprétation autobiographique du texte. Cependant, les critiques sont d'accord sur le fait que la plupart des Essais ont été écrits dans la tour du château de Montaigne. Et comme le lieu de genèse des Essais est connu, il est assez facile de reconstruire la situation concrète dans laquelle Montaigne travaillait à son manuscrit. Dans ce contexte, un détail spatial saute aux yeux : dans les solives du cabinet de travail sont gravées de nombreuses citations de poètes et de philosophes latins, et il semble que Montaigne en changeait souvent en y gravant successivement d'autres citations. Cette appropriation personnelle du lieu de retraite à travers un acte d'inscription est, d'une part, très révélatrice en ce qui concerne l'idée d'une conquête du lieu de retraite qui ne peut s'élaborer qu'à partir d'un acte individuel; d'autre part, ce geste est repris au XIX ${ }^{\mathrm{e}}$ siècle, comme nous le verrons, par Stendhal.

Le lieu idyllique, l'otium et l'introspection sont enfin unis, de façon très programmatique, dans les Rêveries du promeneur solitaire de Jean-Jacques Rousseau. La superposition des trois éléments est surtout reflétée dans la «Cinquième promenade» où il est question du séjour sur l'île Saint-Pierre sur le lac de Bienne en Suisse ${ }^{\mathrm{ro}}$. D'après ce que raconte le je narrant dans la «Cinquième

8. M. de Montaigne, Les Essais, éd. J. Balsamo, M. Magnien et C. Magnien-Simonin, Paris, Gallimard, 2007, p. 29.

9. Ibid., p. 30.

Io. L'île Saint-Pierre constituait, on le sait bien, l'asile de J.-J. Rousseau persécuté entre le I2 septembre 1765 et le 25 octobre 1765 , et ce refuge devient, dans la narration autobiographique, un lieu de recueillement idéal où le sujet s'abandonne à la rêverie et où il se « dévoue à l'oisiveté». 
promenade», les deux mois vécus dans ce lieu «compt[ent] [...] pour le temps le plus heureux ${ }^{\mathrm{II}}$ » de sa vie et dans ce lieu, les "qualités les plus élémentaires de l'idylle [se retrouvent] : la focalisation de l'espace, la beauté de la nature, l'absence de problèmes, la sécurité, l'absence de conflits, les formes d'une vie simple, le loisir nécessaire à des activités créatives et plaisantes $[\ldots]^{12} »$. Que Rainer Warning puisse intégrer si naturellement le loisir dans la conception de l'idylle, nous montre une fois de plus que les idylles sont très souvent considérées comme des lieux de loisir et c'est à travers cette interprétation que la relation entre le loisir et le lieu arcadien a pu devenir un topos puissant de la littérature. Mais alors que l'oisiveté idéale, qui permet au sujet de se consacrer à lui-même, ne reste qu'un désir esquissé chez Montaigne, une telle oisiveté trouve son accomplissement total dans la "Cinquième promenade». Le texte de Rousseau peut donc être considéré comme un texte clé parce qu'il évoque la liaison harmonieuse et réussie entre le loisir et la réalisation de soi à travers l'écriture dans un lieu approprié, même si la "Cinquième promenade" présente moins l'idée d'une écriture dans un moment de loisir que l'idée d'un sujet qui jouit d'un loisir vécu dans le passé, et qui se sert du souvenir de ce loisir pour amplifier son expérience grâce à l'écriture ${ }^{\mathrm{r} 3}$.

La liste des exemples littéraires présentant une association entre loisir et lieu et montrant un sujet profitant du lieu pour en faire la base de son introspection, pourrait facilement être élargie à différentes époques et langues. Même s'il n'est pas possible de prouver dans cet article à quel point cette corrélation est répandue, on peut au moins en citer quelques exemples incontournables : des séjours à la campagne et des promenades dans De vita solitaria de Pétrarque, le fameux «Ich saz ûfeime steine» dans le Reichston de Walther von der Vogelweide, la grotte d'amour dans le Tristan de Gottfried von Straßburg où le couple malheureux commence à raconter des histoires d'amour, mais aussi la petite montagne où se retire la «brigade» dans le Décaméron de Boccace. Pour la littérature de la Renaissance, on peut se référer à l'Arcadia de Jacopo Sannazaro ; dans ce texte, qui est l'archétype du roman pastoral, la fonction protectrice du lieu idyllique est déjà en partie déconstruite et le texte montre les difficultés de la retraite qui échoue si le sujet ne parvient pas tout à fait à exclure de son

(J.-J. Rousseau, Les Rêveries du promeneur solitaire, dans Euvres complètes, éd. B. Gagnebin et M. Raymond, vol. I, Paris, Gallimard, I959, p. I042.)

II. Ibid.

I2. R. Warning, Heterotopien als Räume ästhetischer Erfahrung, Munich, Fink, 2009, p. 43. (Je traduis.)

13. En ce qui concerne l'enrichissement de l'expérience à l'aide de l'imagination, voir aussi l'interprétation de la "Cinquième promenade» par R. Warning (ibid., p. 43-62). 
refuge ce qui le tourmentait dehors. Comme l'essai de Montaigne, l'Arcadia montre à quel point une retraite satisfaisante dépend de la disposition mentale du sujet qui se retire. En somme, on peut constater que les lieux de retraite évoqués dans la littérature européenne sont, jusqu'au XIX ${ }^{\mathrm{e}}$ siècle, des endroits caractéristiques, comme dans Oberman d'Étienne Pivert de Senancour où le héros se retire dans les Alpes suisses ou Walden, œuvre autobiographique de Henry David Thoreau, où la solitude des forêts est un parfait lieu de contemplation. Ces stéréotypes commencent toutefois à changer au début du $\mathrm{Xx}^{\mathrm{e}}$ siècle lorsque les auteurs se mettent à traiter la tradition des idylles avec plus de liberté. Ils s'approprient le modèle bien connu et proposent une variation productive en intégrant aussi dans le paradigme des lieux de retraite des lieux atypiques, lieux que Michel Foucault définira plus tard comme des «hétérotopies ${ }^{\mathrm{T}}$ ». La maison de santé dans $\grave{A}$ la recherche du temps perdu de Marcel Proust, le sanatorium dans Der Zauberberg de Thomas Mann ou l'hôpital psychiatrique dans Die Blechtrommel de Günter Grass illustrent parfaitement ce phénomène.

Même si ce bref passage en revue n'énumère que quelques exemples, on peut repérer des éléments caractéristiques qui ont largement marqué l'idée du lieu de retraite, de loisir et d'autoconstitution du sujet jusqu'à la fin du XIX ${ }^{\mathrm{e}}$ siècle : les lieux sont idylliques, ce sont des lieux solitaires et naturels qui offrent au sujet un éloignement de la société tout en lui ouvrant une vaste perspective. Pour que le sujet retiré puisse accéder plus facilement à des états d'esprit réflexifs, parmi lesquels on peut compter le loisir, la contemplation, la rêverie ou la méditation, les qualités du lieu doivent correspondre aux besoins de tels états d'esprit. Voilà pourquoi les lieux sont typiquement limités dans l'espace, structurés - par un centre et une périphérie, par exemple - et pourquoi ils permettent au sujet de bénéficier d'une vue à la fois variée et sélective. Correspondent à ce paradigme le jardin, la colline, la forêt, des contrées champêtres, mais aussi des maisons ou des chambres qui offrent au sujet la possibilité d'une retraite, l'expérience de la solitude et en même temps un espace libre pour l'accomplissement de soi. Le besoin de l'homme de trouver des lieux de repos lui permettant de se retirer des structures et des contraintes habituelles se reflète dans la littérature de façon très récurrente à travers les époques.

14. M. Foucault, Les Hétérotopies. Radio France, 7 décembre 1966. Version bilingue, traduite par M. Bischoff, Francfort-sur-le-Main, Suhrkamp, 2005. 


\section{Le lieu de retraite et la mémoire du lieu}

Toutes ces remarques mènent à la proposition suivante : un lieu idyllique, grâce à son isolement et à sa beauté, permet au sujet qui s'y retire de faire l'expérience du loisir au sens d'une expérience spatio-temporelle ${ }^{\text {t5 }}$ exceptionnelle, laquelle donne lieu à un mouvement d'introspection et d'autonarration. La littérature nous enseigne, premièrement, qu'il y a des lieux qui sont extrêmement propices à l'expérience du loisir et deuxièmement, que ces lieux sont susceptibles de permettre l'autonarration. Mais bien que ce rapport entre l'expérience de loisir, le lieu idyllique et l'introspection narrative soit prépondérant, il faut se demander si les lieux en question ne disposent pas de qualités plus spécifiques que celles de l'idylle et si ces qualités permettent éventuellement de saisir plus précisément la notion de lieu de retraite.

Avant de raconter ma vie au couvent, ne dois-je pas décrire un peu le couvent?

Les lieux qu'on habite ont une si grande influence sur les pensées qu'il est difficile d'en séparer les réminiscences ${ }^{16}$.

Cette phrase, tirée d'Histoire de ma vie, l'autobiographie monumentale de George Sand, exprime d'une façon très dense à quel point le lien entre le je narrant et les lieux dont il se souvient est important pour l'autonarration. Les lieux n'influencent pas seulement la pensée du je narrant, mais ils sont aussi, de manière inséparable, liés à la mémoire du sujet. On peut même dire que ce sont principalement les lieux qui constituent la mémoire autobiographique d'un je narrant. La mémoire autobiographique des lieux semble être décisive pour l'autonarration, parce qu'elle en forme la trame concrète sur laquelle le sujet peut s'appuyer. Cette mémoire des lieux est présentée dans

15. La perception temporelle du sujet qui vit un moment de loisir ou bien d'otium, ainsi que la représentation narrative de cette expérience temporelle dans l'autobiographie, ne peuvent être examinées ici. Pour l'analyse de cette relation très spécifique, on peut toutefois évoquer la thèse de doctorat de l'auteure qui est en cours d'élaboration (à paraître en 20I7). En ce qui concerne la relation entre l'otium et le temps en général, voir la revue culturelle et littéraire de Tewes : J. Tewes, «Nichts Besseres zu tun. Über Muße und Müßiggänger », dans J. Tewes (dir.), Nichts Besseres zu tun. Über Muße und Müßiggang, Oelde, Tewes, I989, p. 43-60. Pour une perspective psychologique sur ce sujet, voir : M. Wittmann, Gefühlte Zeit. Kleine Psychologie des Zeitempfindens, Munich, Beck, 20I2. G. Figal examine les caractéristiques spatiotemporelles du loisir d'un point de vue philosophique, voir G. Figal, "Muße als Forschungsgegenstand", dans Muße. Ein Magazin, Fribourg-en-Brisgau, 2015 (http://mussemagazin.de/?p=530). Voir également G. Figal, "Die Räumlichkeit der Muße», dans B. Hasebrink et P. Ph. Riedl (dir.), Muße im kulturellen Wandel. Semantisierungen, Ähnlichkeiten, Umbesetzungen, Berlin-Boston, de Gruyter, 20I4, p. 26-33.

I6. G. Sand, Histoire de ma vie 3-4, Genève, Slatkine Reprints, I980, p. 85. 
les textes d'introspection comme un élément de permanence qui s'oppose à la contingence de la mémoire.

Pour préciser ce que j'entends ici par lieu de retraite et par mémoire du lieu, il est nécessaire de mettre ces deux termes en corrélation. Pour ce faire, il faut avant tout poser une question cruciale : comment un lieu idyllique quelconque peut-il se transformer en un lieu de retraite où un je narrant peut parler de lui-même tranquillement? Ce lieu n'est-il qu'un lieu idyllique ou est-il marqué par d'autres caractéristiques? Selon mon hypothèse, il y a une différence importante entre l'idylle ou "l'hétérotopie littéraire», comme l'a définie Rainer Warning ${ }^{17}$, et le lieu de retraite qui permet à un sujet de réfléchir sur lui-même et qui rend possible l'autonarration. Cette différence se manifeste dans un acte de confrontation et d'appropriation. Pour qu'un lieu puisse devenir un lieu de retraite authentique où un sujet peut parler tranquillement de lui-même suivant l'idéal de l'otium, qui permet la connaissance de soi-même, il faut que le sujet s'approprie le lieu en question à travers un effort mémoriel. Le je narrant, qui veut se présenter comme un sujet retiré profitant de l'otium, doit d'abord se confronter à la mémoire du lieu dans lequel il se retire, car l'acte d'appropriation provient d'une confrontation entre les souvenirs du sujet retiré et la mémoire du lieu en question.

En ce qui concerne la mémoire du lieu, je la définis ainsi : au sens strict, le lieu lui-même ne peut pas avoir une mémoire propre; on ne peut parler de mémoire qu'en se référant à des sujets, à des êtres vivants. Pourtant, on peut attribuer à un lieu une fonction de mémoire. À travers la mémoire collective ou individuelle d'un lieu, ce lieu peut gagner, peu à peu, une mémoire du lieu, et cette mémoire du lieu représente des contenus pour un collectif ou un individu. Tandis que la célèbre notion de «lieux de mémoire» établie par Pierre Nora ${ }^{18}$ se réfère à la fonction créatrice d'identité des lieux de mémoire pour la mémoire collective, ou bien la mémoire nationale, il importe de souligner que certains lieux remplissent une fonction de mémoire personnelle. Comme les grandes théories de lieu s'intéressent surtout aux questions de mémoire collective, il est d'autant plus important de mettre l'accent sur le fait qu'il peut y avoir un lieu qui représente une certaine mémoire du lieu pour un seul individu. Par exemple, des lieux comme Waterloo ou Auschwitz ont acquis une fonction propre de mémoire, une mémoire du lieu en relation à une collectivité; par contre, un banc dans un parc de Londres ne dispose d'une mémoire du lieu que par rapport à un seul individu, qui a attribué une certaine fonction mémorielle à ce banc ${ }^{19}$.

I7. Voir R. Warning, Heterotopien als Räume ästhetischer Erfahrung, ouvr. cité, p. 29.

I8. Voir P. Nora, M. Agulhon et P. Boutry, Les Lieux de mémoire, Paris, Gallimard, 1984-1992.

19. Je me réfère ici aux épisodes des Mémoires d'outre-tombe qui se déroulent dans le parc de Kensington à Londres. Voir surtout le livre VI dans F.-R. de Chateaubriand, Mémoires d'outretombe, édition nouvelle, établie d'après l'édition originale et les deux dernières copies du texte, 
Avant qu'un lieu puisse être compris comme un lieu de retraite, il faut donc que le sujet se confronte à la mémoire du lieu et cela veut dire que le sujet crée une relation de réflexion très forte avec son propre lieu de retraite. La confrontation mémorielle avec le lieu de retraite est alors la conditio sine qua non pour l'élaboration de la notion. La mise en avant de l'acte mémoriel permet, de plus, de développer davantage l'idée d'une "hétérotopie littéraire» selon Warning, qui part du principe que «chaque homotopie peut muter et se transformer en hétérotopie si les qualités de l'espace et la disposition affective du sujet convergent vers une telle interprétation ${ }^{20} »$. Le lieu de retraite, en revanche, n'est proprement pas un lieu quelconque. D'après mon hypothèse, il n'est pas possible que chaque lieu puisse devenir un lieu de retraite, et ce n'est ni la qualité de l'espace ni la "disposition affective» du sujet qui détermine le résultat de façon indépendante. Le sujet doit plutôt à chaque fois découvrir s'il peut s'approprier le lieu choisi à travers une confrontation mémorielle ou si cette appropriation lui est impossible. Les raisons pour lesquelles cette appropriation peut échouer sont nombreuses et il faut toujours les analyser séparément et en détail. Le lieu de retraite est alors défini comme une notion qui, en prenant en compte les différentes exigences qu'un sujet formule à l'égard de son lieu de retraite, sous-tend un processus actif d'interaction du sujet avec le lieu choisi. Après avoir circonscrit la relation entre mémoire du lieu et lieu de retraite, il faut à présent montrer comment un tel acte d'appropriation se réalise dans la littérature, ce que je ferai en analysant l'incipit ${ }^{21}$ de la Vie de Henry Brulard de Stendhal.

avec une introduction, des variantes, des notes, un appendice et des index par M. Levaillant et G. Moulinier, Paris, Gallimard, I946-1951.

20. R. Warning, Heterotopien als Räume ästhetischer Erfahrung, ouvr. cité, p. 29. (Je traduis.)

2I. En ce qui concerne l'épineuse question de savoir quel incipit Stendhal aurait finalement choisi pour une publication éventuelle de la Vie de Henry Brulard, je renvoie à l'introduction de l'édition diplomatique où le travail de réécriture de Stendhal est soigneusement expliqué : Stendhal, Vie de Henry Brulard. Écrite par lui-même, édition diplomatique du Manuscrit de la bibliothèque de Grenoble, présentée et annotée par G. Rannaud, transcription établie par G. et Y. Rannaud, Paris, Klincksieck, I996, p. I-LXIII, ici p. XIV-Xv. La version diplomatique est entièrement numérisée, voir <http://stendhal.bm-grenoble.fr/edition_diplomatique/>. Mon analyse va cependant porter sur l'incipit célèbre qui met en scène le protagoniste sur le mont Janicule de Rome et qui figure aujourd'hui comme incipit aussi bien dans l'édition diplomatique que dans l'édition de la Pléiade. 


\section{Rome et une petite colline anodine}

La Vie de Henry Brulard ${ }^{22}$ commence avec une localisation spatiotemporelle très précise du je narrant ${ }^{23}:$ "Je me trouvais** ce matin, I6 octobre I832, à San Pietro in Montorio, sur le mont Janicule, à Rome; il faisait un soleil magnifique.» ( $H B$, p. 529) Le lieu aussi bien que le temps de la narration sont clairement indiqués; le je narrant se trouve en un lieu de retraite qui ne pourrait guère être plus canonique, puisque le Janicule offre une vue magnifique sur l'une des villes à la mémoire la plus riche. Pourtant, cette première phrase est ambivalente et les coordonnées indiquées sont chimériques. En ce qui concerne la situation temporelle, la date du I6 octobre I832 que le je narrant évoque dans le texte, contraste avec la datation que Del Litto propose dans le paratexte de l'édition de la Pléiade : «** 26 oct[obre I8]35. - 23 nov[embre I8]35» (ibid.). Les éditeurs de l'édition diplomatique de la Vie de Henry Brulard transcrivent la note de Stendhal différemment : «I6 oct. 3 I - 23 nov. $35^{24}$ ». Ils plaident pour une datation fictive du texte : on sait en effet que Stendhal a travaillé à son manuscrit entre l'automne 1835 et le printemps $1836^{25}$. Comme il est impossible de déchiffrer avec certitude la note de Stendhal, il n'y a aucun moyen de savoir si le temps de la genèse indiqué est «I83I-I835" ou bien "I835». Ce qui importe pourtant, c'est que la première phrase du texte se réferre déjà à l'âge du je narrant qui est évoqué quelques phrases plus tard. Le je narrant y parle de son cinquantième anniversaire qui approche et comme Stendhal est né en janvier 1783 , cette datation peut être interprétée comme une référence destinée à renforcer l'impression d'authenticité. En même temps, cette première datation nous montre que la Vie de Henry Brulard vit essentiellement des tensions et

22. Stendhal, Vie de Henry Brulard, dans CEuvres intimes II, éd. V. Del Litto, Paris, Gallimard, 1982, p. 525-959. (Désormais HB.) De plus, la forme de citation de l'édition de la Pléiade, où les notes de Stendhal sont marquées par un astérisque, est ici adoptée.

23. Les termes «je narrant» et "je narré» sont utilisés dans l'analyse selon la définition de Gérard Genette (Figures III, Paris, Seuil, 1972, p. 259).

24. Stendhal, Vie de Henry Brulard. Écrite par lui-même, édition diplomatique du Manuscrit de la bibliothèque de Grenoble, ouvr. cité, p. 38.

25. Certes, la Vie de Henry Brulard est parsemée de datations, mais on ne peut pas prendre ces datations comme des faits fiables et authentiques puisqu'il ne s'agit effectivement pas d'un journal intime, mais d'un texte littéraire qui joue avec le temps de la narration et avec l'effet d'une datation authentique, qui est pourtant un élément de stylisation. La genèse de la Vie de Henry Brulard peut donc être reconstruite surtout sur la base du journal de Stendhal; il y a à ce sujet un consensus général de la critique. 
des écarts entre le texte principal et le paratexte ${ }^{26}$. Le cadre spatio-temporel qui est décrit dans la première phrase est ensuite confirmé par des références à l'histoire culturelle associée à l'espace qui entoure le sujet. Après cela, le je narrant, qui se trouve encore sur les escaliers de San Pietro, décrit d'une façon un peu plus détaillée ce qu'il voit :

Ce lieu est unique au monde, me disais-je en rêvant, et la Rome ancienne malgré moi l'emportait sur la moderne, tous les souvenirs de Tite-Live me revenaient en foule. [...] Quelle vue magnifique! C'est donc ici que La Transfiguration de Raphaël a été admirée pendant deux siècles et demi. [...] Ah! Dans trois mois j'aurai cinquante ans; est-il bien possible? ( $H B$, p. 53I)

Rome représente avant tout ici, et la critique l'a déjà montré, «un brouillage des frontières temporelles» ("a blurring of time barriers ${ }^{27}$ ") et elle éveille chez le sujet, qui est en train de contempler la ville et la mémoire de celle-ci, le désir de laisser, lui aussi, des traces, en commençant à raconter son histoire et en puisant dans sa propre mémoire ${ }^{28}$. La pérennité de la ville et de l'art qui y subsiste depuis des siècles contraste avec le caractère éphémère du sujet; ce décalage motive en lui la décision de se rendre compte de la signification de sa propre existence dans une situation d'otium typique.

Je me suis assis sur les marches de San Pietro, et là, j’ai rêvé une heure ou deux à cette idée. Je vais avoir cinquante ans, il serait bien temps de me connaitre. Qu'ai-je été? que suis-je? En vérité, je serais bien embarrassé de le dire. ( $H B$, p. 532)

Le je narrant s'attarde à la contemplation de sa propre temporalité jusqu'au crépuscule et après être rentré chez lui, il grave un mémento dans sa ceinture :

26. Le manuscrit de la Vie de Henry Brulard se trouve à la bibliothèque de Grenoble. Malheureusement, la façon dont Stendhal aurait voulu présenter tous les autocommentaires, les ajouts et les annotations, qui marquent essentiellement la structure et la forme du texte tel qu'il se présente à nos yeux aujourd'hui, n'est pas du tout claire, parce que le texte est resté à l'état de brouillon. Cependant, on peut affirmer que les ajouts, même s'ils se trouvent dans un texte fragmentaire dont la forme définitive ne peut pas être connue, ne sont pas seulement très nombreux, mais qu'en outre, ils ont un caractère métapoétique. Or, comme l'ironie de la Vie de Henry Brulard résulte surtout de la tension entre ces ajouts et la narration autobiographique, on ne peut les laisser à part dans une édition critique. (En ce qui concerne l'histoire de l'édition très compliquée de la Vie de Henry Brulard qui a été publiée la première fois en 1890 dans une version très modifiée, et qui est parue pour la première fois avec les ajouts et les notes de Stendhal en 1949, voir V. Del Litto, "Vie de Henry Brulard. Notice», dans Euvres intimes II, Paris, Gallimard, I982, p. I307-I313.) L'analyse qui suit prendra en compte le texte tel qu'il est présenté au lecteur dans l'édition de la Pléiade de I982. On a choisi cette édition parce qu'elle constitue, malgré l'effort énorme de reconstitution révélé dans l'édition diplomatique, la version la plus facile à lire.

27. J. D. Cremin, Selfhood, Fiction, \& Desire in Stendhal's "Vie de Henry Brulard" \& "Armance", New York, Francfort-sur-le-Main, Berlin, Lang, 1998, p. 23.

28. Voir ibid. 
"I6 octobre I832. Je vais avoir la cinquantaine» (HB, p. 533). Ainsi, la longue rêverie sur le Janicule a d'abord seulement comme résultat la conclusion que l'âge de 50 ans va bientôt arriver, même si cette découverte est notée dans sa ceinture, c'est-à-dire très près du corps. Ce n'est que plus tard dans la même journée, après que le je narrant est rentré seul d'une soirée, qu'il commence vraiment à formuler sa décision d'écrire son autobiographie :

Le soir, en rentrant assez ennuyé de la soirée de l'ambassadeur, je me suis dit : "Je vais écrire ma vie, je saurai peut-être enfin, quand cela sera fini dans deux ou trois ans, ce que j'ai été, gai ou triste, homme d'esprit ou sot, homme de courage ou peureux, et enfin au total heureux ou malheureux, je pourrai faire lire ce manuscrit à Di Fiore."

Cette idée me sourit. Oui, mais cette effroyable quantité de Je et de Moi! Il y a de quoi donner de l'humeur au lecteur le plus bénévole. Je et Moi, ce serait, au talent près, comme M. de Chateaubriand, ce roi des égotistes. ( $H B$, p. 533)

Le negotium "ennuyeux" de la soirée chez l'ambassadeur contraste ici clairement avec le projet excitant de raconter sa vie. En même temps, la demande formulée dans cette entreprise est précisée : l'écriture de sa vie doit tout d'abord servir à atteindre une connaissance approfondie de soi-même, car celle-ci n'a pas encore été acquise. En apparence, le texte semble vouloir présenter une narration harmonieuse qui intègre des expériences disparates et contingentes dans un ensemble bien agencé. Cette confession traditionnelle d'un je narrant qui a l'air de vouloir écrire un texte qui répond à un impératif téléologique, semble convenir à la conception théorique de l'autobiographie telle qu'elle a été présentée, notamment par Georg Misch et Wilhelm Dilthey au début du $\mathrm{xx}^{\mathrm{e}}$ siècle. Car, selon Dilthey, la «biographie du soi [est] [...] la forme la plus effective et la plus instructive dans laquelle la compréhension de la vie se présente à nous ${ }^{29} »$. Et Georg Misch, qui a marqué la définition du genre par une simple décomposition du mot - l'autobiographie est «la description (graphia) de la vie (bios) d'un seul individu par lui-même (auto) $)^{30}$ » parle aussi d'une "fécondité» spécifique de l'autobiographie qui résulte de la "diversité des formes $\gg^{3 \mathrm{I}}$ qu'elle peut offrir. Cependant, la décision d'écrire sa vie formulée dans l'incipit de la Vie de Henry Brulard ne fait effectivement que semblant

29. W. Dilthey, «Das Erleben und die Selbstbiographie», dans G. Niggl (dir.), Die Autobiographie. Zu Form und Geschichte einer literarischen Gattung. 2., um ein Nachwort zur Neuausgabe und einen bibliographischen Nachtrag ergänzte Auflage, Darmstadt, Wissenschaftliche Buchgesellschaft, I998, p. 2I-32, p. 28. (Je traduis.)

30. G. Misch, «Begriff und Ursprung der Autobiographie», dans G. Niggl (dir.), Die Autobiographie. Zu Form und Geschichte einer literarischen Gattung. 2., um ein Nachwort zur Neuausgabe und einen bibliographischen Nachtrag ergänzte Auflage, Darmstadt, Wissenschaftliche Buchgesellschaft, I998, p. 33-54, p. 38. (Je traduis.)

3I. Ibid., p. 37. 
d'être une décision téléologique, car la dimension métaréflexive du texte se révèle déjà dans le même paragraphe. Le je narrant ne prévoit pas seulement une production de texte qui prendra «deux ou trois ans» (HB, p. 533), mais il problématise aussi les difficultés formelles de son projet, qui résultent surtout de la redondance infinie de la première personne ${ }^{32}$. En outre, il anticipe les attentes de ses lecteurs et, par ce geste, prend explicitement ses distances avec Chateaubriand, ce "roi des égotistes" (ibid.) dont l'excessive mise en scène de soi est ici, tout comme plus tard dans le texte, ouvertement critiquée ${ }^{33}$. En disant ensuite qu’on doit présenter, même au lecteur «bénévole» (ibid.), quelque chose d'amusant, le narrateur signale au lecteur implicite qu' il connait bien les règles rhétoriques. En un seul paragraphe, le je narrant fait donc part de sa décision d'écrire sa vie, problématise des questions esthétiques à propos de la production et de la réception de l'œuvre et s'ancre lui-même de façon critique dans la tradition autobiographique. Mais bien que la décision de commencer le récit de sa vie soit dès lors clairement communiquée, le je ne la met pas tout de suite en acte. Au contraire, un délai irritant de trois ans est évoqué concernant "l'heure où il écrit ${ }^{34}$ » :

Je ne continue que le 23 novembre I835. La même idée d'écrire my life m'est venue dernièrement pendant mon voyage de Ravenne; à vrai dire, je l'ai eue bien des fois depuis 1832, mais toujours j'ai été découragé par cette effroyable difficulté des $J e$ et des $M o i$, qui fera prendre l'auteur en grippe, je ne me sens pas le talent pour la contourner. $(H B$, p. 534)

Trois ans après avoir eu, en rêvant sur les marches de San Pietro, pour la première fois l'idée d'écrire sa vie, le je narrant admet qu'il est encore occupé par les mêmes problèmes formels et les mêmes questions de véracité («mais combien ne faut-il pas de précautions pour ne pas mentir!», $H B$, p. 537) et il dément, dans un appel direct au lecteur, un premier événement biographique déjà raconté : «non, mon lecteur, je n'étais point soldat à Wagram en I809" (ibid.). Certes, le premier chapitre veut nous présenter, selon les règles de l'exposition, les raisons pour lesquelles l'autobiographie est entreprise, mais

32. Dans le paragraphe suivant, le je narrant envisage d'écrire sa vie à la troisième personne, mais il doute tout de suite de cette idée en disant : "mais comment rendre compte des mouvements intérieurs de l'âme?» ( $H B$, p. 535)

33. Le je narrant utilise Chateaubriand dans plusieurs épisodes comme un contre-modèle dont il veut se distinguer. Voilà pourquoi il souligne par exemple au chapitre xxiv qu'il ne craint pas, comme Chateaubriand ou Villemain le font, d'être sifflé pour ce qu'il raconte : "Je ne suis ni timide ni mélancolique en écrivant et m'exposant au risque d'être sifflé; je me sens plein de courage et de fierté quand j'écris une phrase qui serait repoussée par l'un de ces deux géants (de I835), MM. de Chateaubriand ou Villemain.» (HB, p. 767)

34. J. Starobinski, «Le style de l'autobiographie», Poétique, n 3, I970, p. 257. 
le vrai commencement de la narration est sans cesse différé. Comme je l'ai déjà indiqué, la première phrase de l'incipit ("Je me trouvais** ce matin, I6 octobre I832, à San Pietro in Montorio, sur le mont Janicule, à Rome; il faisait un soleil magnifique.", $H B$, p. 529) qui semble être tellement précise, n'est pas du tout fiable : elle se révèle être le prélude à un jeu récurrent qui démonte peu à peu la conception traditionnelle de l'autobiographie.

Mais ce ne sont pas seulement les questions de datation de la genèse du texte et les questions de genre qui restent ambiguës. L'épisode du Janicule est imprécis particulièrement en ce qui concerne l'espace, parce que la colline, ce topos classique qui invite le sujet retiré à s'y contempler, à méditer et à réfléchir sur soi, se révèle, au début du deuxième chapitre, n'être qu'un pseudolieu de retraite. Le vrai lieu de retraite où le je narrant commence à écrire et qu'il s'approprie dans un acte mémoriel se trouve en dehors de Rome, près de Civitavecchia, sur un monticule beaucoup moins important. Certes, le vénérable Janicule sert de modèle topologique pour la décision d'écrire sa vie, mais ce dont la critique n'a pas encore débattu's5, c'est que le vrai lieu de retraite, qui est en même temps celui de la genèse du texte, n'est introduit que de façon subséquente, au deuxième chapitre. Rome, le «lieu de mémoire» monumental et établi, est ainsi opposée à la province sans histoire ${ }^{36}$ et le moment décisif de la genèse du texte autobiographique est déplacé du Janicule vers une colline totalement anodine. En même temps, le texte autobiographique est ainsi séparé de Rome, qui peut être considérée comme un lieu lisible, et peut donc être compris comme une tentative de se soustraire à l'immense mémoire du lieu que représente la ville de Rome. Aleida Assmann, qui revient, dans sa monographie sur les espaces de mémoire et la mémoire collective, sur la promenade de Pétrarque et de Giovanni Colonna à travers Rome, comprend Rome comme un lieu où les mémoires se manifestent de façon bien visible :

Pour les deux promeneurs, le temps se fortifie et se transforme en espace; ce que le temps rend invisible avec ses processus de vol et de destruction, est maintenu de façon mystérieuse dans les lieux. La chronologie devient une topologie de l'histoire qu'on peut parcourir en se promenant et qu'on peut, successivement, déchiffrer sur place ${ }^{37}$.

35. Alors que l'incipit et la vue panoramique sur Rome ont été souvent l'objet de l'analyse, l'exposition retardée du lac d'Albano n'a pas encore été examinée. Voir par exemple J. D. Cremin, ouvr. cité, p. 23-29. Voir aussi C. Lang, "Une autobiographie en dérive», Modern Language Notes, $\mathrm{n}^{\circ}$ 5, 1979, p. 1076-1078.

36. V. Del Litto appelle la petite ville d'Albano, qui se trouve à 40 kilomètres au sud de Rome, une «localité de villégiature» et il ajoute que Stendhal y était souvent pour échapper à la chaleur d'été de Rome. Voir V. Del Litto, "Vie de Henry Brulard. Notice», art. cité, p. I332. 37. A. Assmann, Erinnerungsräume. Formen und Wandlungen des kulturellen Gedächtnisses, Munich, Beck, 2009, p. 3II. (Je traduis.) 
Ce que le je narrant voit quand il se trouve sur le Janicule peut donc, suivant l'idée d'Assmann, être désigné comme une «topologie de l'histoire», ce qui veut dire aussi que Rome fournit une mémoire du lieu dans une perspective collective très riche. Rome est même tellement importante que les processus mémoriels d'attribution sont très répandus et effectifs, de sorte que la ville se charge d'une fonction de mémoire extraordinaire. De plus, la mémoire de Rome représente une intégration réussie des différentes époques et constitue donc un modèle pour le texte autobiographique qui cherche aussi à surmonter des discontinuités temporelles dans l'écriture. Considérant cette fonction de mémoire, la retraite du je narrant dans la Vie de Henry Brulard prend une signification importante puisque le sujet se retire dans un lieu qui n'a pas de fonction de mémoire; cette retraite peut être interprétée comme la tentative de trouver un lieu sans histoire, un lieu qui ne porte pas encore une écriture et où le sujet peut raconter tranquillement et à loisir l'histoire de sa propre vie. L'introduction subséquente d'un lieu de retraite individuel révèle à quel point le premier chapitre est une mise en scène calculée et stylisée qui veut faire croire qu'on lit un incipit autobiographique traditionnel qui évoque les motifs de mémoire et de victoire sur le temps. Mais en fait, l'incipit représente une rupture avec les attentes traditionnelles du lecteur, parce que la narration autobiographique ne commence pas lorsque le je narrant se trouve sur le Janicule et qu'il contemple la ville de Rome, mais lorsqu'il se trouve dans un lieu sans nom. Le je narrant montre qu'il connaît très bien la tradition autobiographique et qu'il pourrait facilement s'y inscrire pour ensuite la démonter de façon ironique :

Mais, l'autre jour*, rêvant à la vie dans le chemin solitaire au-dessus du lac d'Albano, je trouvai que ma vie pouvait se résumer par les noms que voici, et dont j'écrivais les initiales sur la poussière comme Zadig, avec ma canne, assis sur le petit banc derrière les stations du Calvaire des Minori Osservanti bâti par le frère d'Urbain VIII, Barberini, auprès de ces deux beaux arbres enfermés par un petit mur rond.

* Écrit de nuit. (HB, p. 54I)

Comme dans l'incipit, le je narrant évoque de nouveau la rêverie qui se déroule en un lieu élevé, mais cette fois-ci, il entreprend aussi son premier acte d'écriture. Le Janicule constitue alors le lieu de naissance de l'idée d'écrire, mais le lieu idyllique au-dessus du lac d'Albano en province est le lieu de genèse du texte, puisque c'est là que l'écriture devient enfin possible. Les initiales que le je narrant grave avec sa canne dans la poussière sont indiquées par la suite - il s'agit de onze noms de femme - et le lieu de retraite est concrétisé dans la première esquisse introduite dans le texte. 


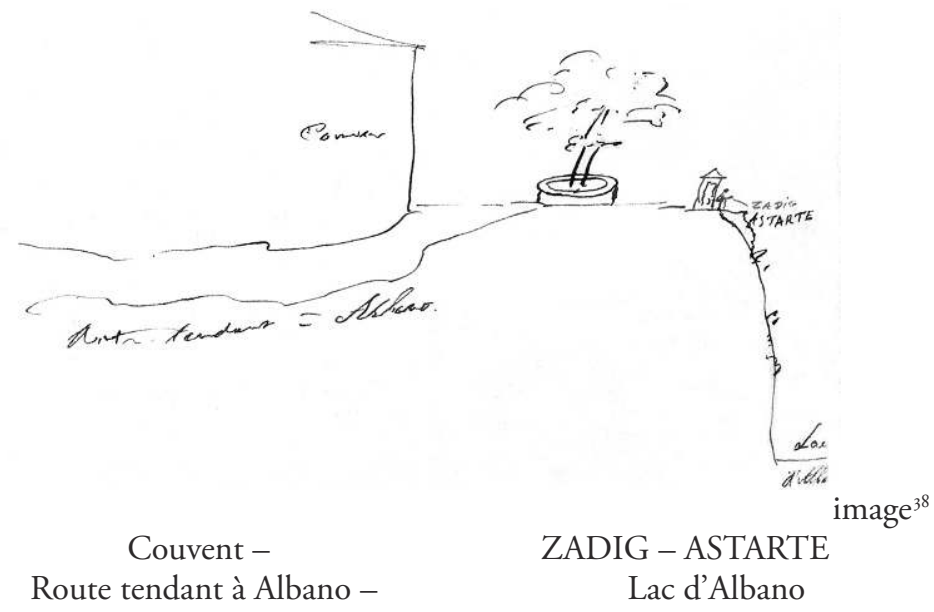

Cette esquisse est une des rares esquisses mimétiques, alors que la plupart des croquis dessinés tout au long du texte restent très schématiques. Le lieu de retraite est donc très précisément mémorisé par le je narrant et il est reconstruit de façon précise non seulement linguistiquement mais aussi graphiquement. Ce lieu qui est décrit d'une manière si détaillée ne possède pas seulement les traits traditionnels du locus amoenus, mais il se présente aussi comme un véritable lieu de retraite, tel qu'il a été défini dans la deuxième partie de cet article (supra). Ce lieu répond au besoin du je narrant de conquérir avec son texte un terrain inconnu et à la nécessité que ce lieu de retraite soit encore vierge.

En ce lieu élevé, le je narrant est assis sur un banc encadré par deux «beaux arbres » qui sont, pour leur part, "enfermés d'un petit mur rond». Là, il grave, perdu dans ses pensées, quelques initiales de noms dans le sol poussiéreux, noms dont il présume qu'ils « résumeraient» toute sa vie. Ainsi, le texte nous présente un sujet qui raconte qu'il se trouve en un lieu idyllique et isolé qui est strictement limité, mais qui permet en même temps, grâce à sa situation en surplomb, une vue panoramique. De plus, le sujet, totalement livré à lui-même, prend sa place dans la tradition littéraire en évoquant Voltaire et arrive à représenter toute sa vie de façon métonymique dans un premier acte d'écriture en gravant dans la poussière les initiales des femmes jadis aimées. Chaque initiale représente alors un épisode différent de sa vie. Ce moment crucial où le sujet réussit pour la première fois à arranger systématiquement les événements de sa vie, et où il trouve,

38. Esquisse contenue dans le manuscrit de la Vie de Henry Brulard. Le manuscrit est conservé à la bibliothèque de Grenoble sous la référence $\mathrm{n}^{\circ} \mathrm{II} 3$, folio 53v. Ici, il est cité d'après : Stendhal, Vie de Henry Brulard, édition diplomatique du Manuscrit de la bibliothèque de Grenoble, ouvr. cité, p. I07. On trouve la transcription de la légende dans l'édition diplomatique sur la même page. 
avec les femmes autrefois aimées, un moyen lui permettant de visualiser sa vie dans une structure chronologique et thématique, peut être considéré comme une mise en scène de l'expérience de l'otium qui n'apparaît qu'en relation avec un lieu spécifique. Là, le sujet est libre de toutes les contraintes de la forme qui se seraient imposées à lui sur le Janicule ${ }^{39}$.

L'opposition fonctionnelle et locale entre le Janicule et la colline située au bord du lac d'Albano est encore une fois soulignée par le je narrant lorsqu'il rappelle, dans une rétrospective, son acte d'écriture entrepris sur cette colline :

Il y a deux mois donc, en septembre I835, rêvant à écrire ces Mémoires, sur la rive du lac d'Albano (à deux cents pieds du niveau du lac), j'écrivais sur la poussière comme Zadig ces initiales :

V. A. A. M.M.A.A.A.M.C.G.A.

I $2 \quad 32 \quad 456^{40}$

(Mme Azur dont j'ai oublié le nom de baptême).

Je rêvais profondément à ces noms, et aux étonnantes bêtises et sottises qu'ils m’ont fait faire (je dis étonnantes pour moi, non pour le lecteur, et d'ailleurs je ne m'en repens pas). ( $H B$, p. 543-544)

Le présent de la narration est ici marqué par la construction temporelle déictique «il y a deux mois» et la datation "en septembre i835", de sorte que le lecteur comprend qu'entre la première idée d'écrire son autobiographie que le je narrant a eue en octobre I832 sur le Janicule et le premier acte d'écriture entrepris près du lac d'Albano, il doit y avoir un laps de temps de presque trois ans. Cette indication temporelle correspond à un souvenir très précis du lieu ("à deux cents pieds du niveau du lac») qui montre encore une fois combien le premier moment de l'écriture, qui rassemble le lieu de retraite, la réflexion sur la mémoire du lieu et l'introspection, s'est profondément gravé dans la mémoire du sujet. De plus, la rêverie, ce motif bien connu depuis Rousseau et Senancour, joue un rôle important dans les deux épisodes contemplatifs, car

39. Il faut préciser que le je narrant prend, plus tard dans le texte, une position ambivalente à l'égard de Civitavecchia et de la tranquillité qui y règne. Il commente par exemple le manque de médias subsidiaires dont il aurait besoin pour compenser sa mémoire lacunaire : "Je n'ai aucun livre à $\mathrm{C}[\mathrm{ivit}] \mathrm{a}-\mathrm{V}$ [ecchi]a pour le chercher [sc. un renseignement sur le parlement de $\mathrm{Pau}$ ], mais tant mieux, ce livre-ci, fait uniquement avec ma mémoire, ne sera pas fait avec d'autres livres.» (HB, p. 9I8) La province n'est alors pas toujours vue comme un lieu de loisir, et le séjour du sujet dans la petite ville peut parfois ressembler à un séjour forcé ou bien à un exil où le je narrant désire revoir les métropoles culturelles comme Rome ou Milan. En ce qui concerne la production réelle du texte, il faut savoir que Stendhal n'a plus écrit une seule phrase de la Vie de Henry Brulard après avoir reçu l'autorisation de quitter son poste de consul à Trieste en mars I836 pour prendre des vacances. Le départ de la province correspond alors, sur le plan biographique, à l'abandon final du texte.

40. V. Del Litto ne défait pas seulement les abréviations des noms, mais il explique aussi que les «chiffres indiquent la succession chronologique» (voir V. Del Litto, «Vie de Henry Brulard. Notice», art. cité, p. 1335). 
c'est dans ce mode que le je narrant, après trois années d'attente, arrive enfin à lancer le projet prévu. Aussi concrètement que le je narrant raconte la naissance de l'écriture, il rappelle de nouveau la naissance de l'idée d'écriture dans une annotation ultérieure. Il note : « ${ }^{*} 29$ j[anvie]r 1836 . Pluie et temps froid. Promenade à San Pietro in Montorio où j'ai eu l'idée d'écrire ceci en I832." $(H B$, p. 863) Il ne rappelle pas seulement la date de sa première promenade sur le Janicule, mais met aussi en avant un contraste climatique : à l'époque, «il faisait un soleil magnifique» ( $H B$, p. 529), dans la situation présente, il fait froid et il pleut. En évoquant ce contraste, le je narrant amplifie ce que la datation indique : une distance décisive qui sépare le je narrant actuel de sa première idée d'écrire sa vie. La deuxième promenade vers l'église de San Pietro ressemble ainsi à un chemin de pèlerinage qui mène le sujet au lieu de naissance de sa propre idée, et cela signale en même temps la dimension séculaire du texte, car ce n'est pas pour penser à Dieu que le je narrant se dirige de nouveau vers l'église de San Pietro, mais pour réfléchir sur lui-même.

Le Janicule et la petite colline près du lac d'Albano sont encore liés par un autre élément, parce qu'il y a, aux deux endroits, des bâtiments religieux derrière le je narrant. Cette localisation du je narrant devant l'église de San Pietro et devant le couvent sur la colline à Civitavecchia est symbolique, car dans les deux épisodes les lieux spirituels sont subordonnés à l'importance du sujet; ils ne constituent qu'un arrière-plan devant lequel le sujet réfléchissant peut se dessiner plus concrètement. Le fond religieux de ces deux épisodes semble rappeler implicitement le schéma augustinien selon lequel l'autobiographie est surtout une confession devant Dieu, mais ce modèle est clairement traité comme quelque chose d'obsolète : le couvent dans l'esquisse n'est qu'une silhouette falote alors que le je écrivant qui se trouve dans la partie droite de l'esquisse est, comme son lieu de retraite, facilement reconnaissable. Le je autonome qui cherche son lieu de retraite individuel et qui se détourne de Rome et de sa mémoire trop chargée, se détourne alors aussi - du moins c'est ce que semblent suggérer les pâles motifs religieux - d'une tradition autobiographique dont la légitimation est chrétienne comme chez saint Augustin ou chez Chateaubriand. Alors que Chateaubriand sacralise son texte et en fait une vraie "cathédrale de mémoire ${ }^{4 \mathrm{I}}$ " dont la construction monumentale est toujours justifiée par sa foi, on trouve, à l'opposé, un programme séculier au début des mémoires de Stendhal.

Le Janicule à Rome invitait le je isolé à contempler l'histoire de la ville et l'histoire culturelle qui s'y est accumulée pendant les siècles, de sorte que le sujet est véritablement obligé de se penser en relation avec ces signes qui indiquent

4I. Voir F.-R. de Chateaubriand, Mémoires d'outre-tombe, ouvr. cité, p. 435-436. 
un passé très lointain. Le sujet n'arrive pas à réfléchir sur lui-même hors de ce contexte, mais il est contraint par la mémoire flamboyante du lieu à faire quelques considérations spécifiques. Par contre, le lieu de retraite individuel que le sujet choisit selon ses besoins lui permet de se concentrer exclusivement sur lui-même. C'est en ce lieu que le sujet peut devenir l'objet de ses propres réflexions. À Rome, il y avait tout un paradigme d'histoire qui envahissait le lieu et qui inhibait la narration autobiographique. Plus loin dans le texte, le je narrant évoque encore une fois - ce qui est caractéristique de la redondance stylistique de la Vie de Henry Brulard - cette première idée d'écrire sa vie qu'il a eue sur le Janicule. Il y déconstruit le concept d'une autonarration authentique d'une façon encore plus virulente qu'auparavant :

Ceci [le fait qu'il ne se souvient plus pourquoi son précepteur détesté, l'abbé Raillane, devait enfin quitter son poste] constitue un défaut de ma tête, dont je découvre plusieurs exemples, depuis trois ans que m'est venue, sur l'esplanade de San Pietro in Montorio (Janicule), l'idée lumineuse que j'allais avoir cinquante ans et qu'il était temps de songer au départ, et auparavant, de se donner le plaisir de regarder un instant en arrière. Je n'ai aucune mémoire des époques ou des moments où j'ai senti trop vivement. [...] Mais je m'égare. La grande difficulté d'écrire ces Mémoires, c'est de n'avoir et de n'écrire juste que les souvenirs relatifs à l'époque que je tiens par les cheveux; par exemple, il s'agit maintenant des temps, évidemment moins malheureux, que j’ai passés sous le maitre Durand. ( $H B$, p. 628)

Le je narrant se plaint ici de sa mémoire défaillante, de l'inévitable digression et du caractère arbitraire de la mémoire qui a pour conséquence que seuls quelques souvenirs peuvent être fixés sur le papier et que d'autres restent dans l'oubli. Il affirme que les moments de sa vie qui sont chargés d'émotions intenses ont disparu de sa mémoire, mais en disant cela, il caricature son propre programme, car le texte raconte effectivement beaucoup d'épisodes relatifs aux états affectifs. De la même manière, les épisodes qui sont intentionnellement passés sous silence, comme la mort traumatisante de la mère, montrent que le sujet pourrait bien les évoquer s'il le voulait. Le je narrant se contredit ainsi explicitement, ce qui montre une fois de plus combien le texte autobiographique de Stendhal vise à s'affranchir des conventions et des limites du genre. Il n'est plus question de reconstruire l'histoire d'une vie de la façon la plus détaillée et la plus véridique possible, mais l'objectif est de témoigner de toutes les difficultés de l'écriture autobiographique et de les reproduire de façon crédible.

Cet objectif comprend aussi la mise en lumière du contraste entre une évaluation très positive du présent et une évaluation négative du passé, telle qu'elle se manifeste dans la dernière citation. Jean Starobinski a proposé une terminologie utile pour cette évaluation dichotomique qu'il a établie en analysant les Confessions de Rousseau. Il distingue un «ton élégiaque» d'un «ton 
picaresque ${ }^{42}$ » le "ton élégiaque» est utilisé pour pleurer le «sentiment du bonheur perdu ${ }^{43}$ » et indique un mécontentement au niveau du présent de la narration. Le "ton picaresque», en revanche, est utilisé pour montrer que le passé est jugé comme un «temps des faiblesses, de l'erreur, de l'errance, des humiliations [et] des expédients ${ }^{44} »$ et que ce passé est désormais surmonté. Dans le texte de Stendhal, on trouve assez souvent des passages caractérisés par le ton picaresque, dont la dernière citation donne un exemple : là, le passé raconté est clairement dévalué par rapport au présent de la narration. Starobinski ajoute une précision :

[...] le narrateur picaresque [...] peut se moquer de l'être obscur et besogneux qui donnait tête baissée dans toutes les illusions du monde. Il parlera donc de son passé avec ironie, condescendance, apitoiement, amusement. Ce ton narratif requiert souvent la présence imaginée d'un destinataire, d'un confident, dont il faut se faire un complice indulgent et amusé, par la vertu de l'enjouement que l'on met à raconter les tours les plus pendables ${ }^{45}$.

Cet aspect de la définition du narrateur picaresque s'applique bien à une grande partie de la Vie de Henry Brulard, car le je narrant, dont l'autodérision est la caractéristique principale, demande toujours pardon à ses futurs lecteurs. Il s'excuse pour son style digressif, se rit souvent de son identité d'autrefois, c'est-à-dire du je narré, et souligne ainsi sa maturité et sa supériorité actuelle. Ce modèle d'un narrateur mûr, qui peut exposer tranquillement toute sa vie passée dans une rétrospective apaisée et qui est capable de se moquer de ses anciennes peines et souffrances semble représenter en même temps le cas classique de la narration autobiographique qui présuppose que le je narrant a atteint un âge digne qui lui permet une introspection distanciée et réfléchie. Or, le je narrant de la Vie de Henry Brulard est véritablement singulier si l'on considère son autodérision permanente et sa déconstruction programmatique qui rompent complètement avec l'idée d'une autonarration authentique ${ }^{46}$.

42. J. Starobinski, «Le style de l'autobiographie», art. cité, p. 264.

43. Ibid.

44. Ibid.

45. Ibid., p. 264.

46. L'impossibilité d'une narration autobiographique authentique est par exemple discutée à travers la métaphore de la «fresque» que le je narrant emploie plusieurs fois. Cette métaphore sert à accentuer le fait que la mémoire du sujet est très fragmentaire et que ses mécanismes complémentaires sont incontrôlables. De plus, l'acte d'écriture est explicitement compris comme la "découverte de quelque chose de nouveau", ce qui montre que le je narrant intègre la dimension romanesque et créatrice de son écriture dans le discours : "À côté des images les plus claires je trouve des manques dans ce souvenir, c'est comme une fresque dont de grands morceaux seraient tombés. Je vois Séraphie se retirant de la cuisine et moi faisant la conduite à l'ennemi le long du passage. [...] Je m'interroge depuis une heure pour savoir si cette scène est bien vraie, réelle, ainsi que vingt autres qui, évoquées des ombres, reparaissent un peu, 
Chez Stendhal, la description de la contingence et de l'autodynamisme de la narration se trouve toujours au centre de l'intérêt. Il en résulte que la présentation de la vie personnelle n'est plus, comme chez Chateaubriand ou chez George Sand, le but primaire de la narration, mais qu'elle est subordonnée à la discussion du processus de l'écriture.

On voit donc bien que la Vie de Henry Brulard commence avec le geste traditionnel d'un sujet avisé qui attend de son autonarration qu'elle lui offre une nouvelle connaissance plus profonde de lui-même, mais le je narrant montre tout de suite qu'il est conscient de l'aporie de cette entreprise. Bien qu'il sache qu'une autoprésentation véridique et authentique est impossible et bien qu'il connaisse le caractère extrêmement construit de l'autobiographie, il s'engage dans une confrontation avec sa mémoire défectueuse. Le vrai commencement de cette narration qui discute surtout ses propres difficultés n'est pourtant possible qu'en un lieu de retraite qui ne soit pas déjà sur-écrit de mémoires. Il lui faut un lieu qui en est affranchi et qu'il peut s'approprier facilement. L'appropriation mémorielle de la petite colline anodine en province ne demande pas - comme le fait le Janicule à Rome - que le sujet se confronte à la mémoire imposante d'un lieu. La conquête aisée de la petite colline sans histoire permet au sujet de vivre un moment de loisir pendant lequel il arrive à graver quelques-uns des moments-clefs de sa vie en son lieu de retraite. L'appropriation du lieu de retraite et le premier acte d'écriture en ce lieu constituent donc le moment décisif de l'écriture de soi, qui ne pouvait se réaliser sur le Janicule.

après des années d'oubli.» (HB, p. 644-645) En reprenant la métaphore de la fresque un peu plus tard, le je narrant souligne encore une fois sa chasse de souvenirs précis et datables, voir ibid., p. 657. 